\title{
latindex : HORIZONTE DE CALIDAD EDITORIAL Y VISIBILIDAD CIENTÍFICA
}

\author{
LATINDEX: EDITORIAL QUALITY HORIZON AND SCIENTIFIC VISIBILITY \\ Manuel Huamán-Guerrero 1,a,b,c, Jhony A. De La Cruz-Vargas 2,d,e,f
}

\section{"La ciencia que no se ve, no existe"}

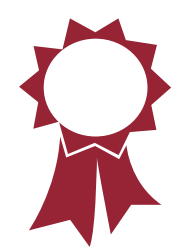

La difusión de los trabajos de investigación científica y tecnológica desarrollados en las universidades e institutos superiores en América Latina, presenta niveles aun no satisfactorios. Algunas universidades cuentan con sus propias revistas científicas que promueven esa difusión, pero en muchas instituciones difundir los trabajos científicos depende exclusivamente de la iniciativa de cada autor. El impacto de estas publicaciones también es muy reducido, aspecto que se viene superando gracias a la conformación de redes regionales. Resulta interesante analizar los principales sistemas regionales de información y catálogos disponibles, como Latindex, Redalyc y Scielo, así como sus características, criterios de calidad, y sus perspectivas futuras.

Las universidades latinoamericanas son las responsables en nuestros países de renovar el saber humano y proporcionar los conocimientos necesarios para orientar el rumbo de la sociedad. Sin embargo, el escaso énfasis dado a la investigación científica es una de las debilidades de estas instituciones, que no siempre ocupan el lugar que les corresponde. Las universidades en la región generan actualmente programas de investigación muy limitados en recursos, autonomía y perspectivas, salvo honrosas excepciones.

Además de la carencia en términos de programas de investigación, se tienen grandes dificultades en la región en cuanto a la difusión de las investigaciones realizadas. La ciencia que no se ve no existe, lema que resume la importancia de esta actividad comunicativa. Para ser validada una investigación por la comunidad científica internacional, necesariamente se debe acceder a ella, ser visible para todos los científicos del mundo. Este paso fundamental, de la producción científica a la comunicación amplia de los resultados, hace la diferencia entre las universidades de primer mundo, y las que no llegan a ocupar puestos de relevancia en la consideración internacional como centros de estudios superiores de excelencia.

Además de contar con el ISSN (International Standard Serial Number) disponible para las revistas en su versión impresa y online, ellas deben ingresar a Programas de información científica indexadas como Latindex, SciELO (Scientific Electronic Library Online), BEIC (Biblioteca Electrónica de Información Científica), entre otros.

Latindex (Sistema Regional de Información en Línea para Revistas Científicas de América Latina, el Caribe, España y Portugal) es un sistema de información académica, sin fines de lucro y de consulta gratuita, especializado en revistas académicas editadas en Iberoamérica; ofrece también información sobre revistas de vocación latinoamericanista editadas fuera de la región. El sistema es fruto de la cooperación entre distintas instituciones de 23 países.

Latindex tiene sus antecedentes en las recomendaciones emanadas del Primer Taller sobre Publicaciones Científicas en América Latina, celebrado en 1994 en Guadalajara, México, donde se puso claramente de manifiesto la falta de un sistema de información propio para las revistas de carácter científico y académico de los países de habla castellana y portuguesa.

Latindex comenzó a gestarse en la Universidad Nacional Autónoma de México (UNAM), hacia finales de 1995. En su diseño se planteó como elemento indispensable del sistema que éste tuviera un carácter regional y cooperativo, que, al no estar centralizado en una determinada institución o país, basara su fortaleza en el trabajo

\footnotetext{
'Vicerrector Académico de la Universidad Ricardo Palma.

a Director General del INICIB.

bEditor en Jefe de la Revista de la Facultad de Medicina Humana.

c Doctor en Medicina y Especialista en Cirugía Oncológica.

${ }^{2}$ Director Adjunto del INICIB.

Coeditor de la Revista de la Facultad de Medicina Humana.

e Doctor en Medicina, Maestría en Investigación Clínica.

Especialista en Oncología Médica.
}

Citar como: Manuel Huamán-Guerrero, Jhony A. De La Cruz-Vargas. Latindex: Horizonte de calidad editorial y visibilidad científica [Editorial]. Rev. Fac. Med. Hum. 2016;16(3):7-10. DOI 10.25176/RFMH.v16.n3.645 
compartido y en la cercanía a las fuentes que generan o distribuyen datos sobre las revistas. La reunión de instalación de la red Latindex se llevó a cabo en la UNAM en febrero de 1997. La Primera versión del proyecto fue de alcance latinoamericano y tuvo como nombre Índice Latinoamericano de Publicaciones Científicas Seriadas. Con la incorporación en 1998 de España y Portugal, el sistema cambió a su actual denominación y cobertura.

Latindex ofrece cuatro bases de datos sobre las revistas académicas que se publican en Iberoamérica:

\section{Directorio}

Disponible desde 1997, registra la existencia de revistas con contenido académico editadas en los países de la región. Cada registro ofrece los datos básicos de identificación tales como título, año de inicio, institución editora, disciplina de especialización, tiraje, precio y datos de contacto. Para octubre de 2012 había 21,000 revistas registradas (vigentes o cesadas) publicadas en 32 países y territorios. Incluye además revistas editadas por organismos internacionales donde participan los países de Iberoamérica, como la Organización de los Estados Americanos (OEA), Organización de Estados Iberoamericanos para la Educación, la Ciencia y la Cultura (OEI) o el Instituto Panamericano de Geografía e Historia (IPGH), entre otros. Integra, asimismo, bajo la denominación de "Latinoamericanistas" aquellas revistas con contenidos sobre la región, pero que son editadas en países europeos.

\section{Catálogo}

Disponible desde 2002, es un subconjunto de las revistas incluidas en el Directorio. Forman parte del Catálogo solamente aquellas revistas que cumplen con un umbral de características editoriales establecidas por el Sistema mediante la aplicación de criterios de calidad editorial. Estos criterios están disponibles para consulta en el propio sitio web de Latindex. El Catálogo no fue creado como un instrumento de evaluación propiamente dicho, sino como un servicio de referencia especializado en la calidad editorial de cada revista. Para octubre de 2012, el catálogo registra más de 6400 títulos.

\section{Revistas electrónicas}

También disponible desde 2002, permite la localización automática de las publicaciones incluidas en el Directorio, que tienen una versión en línea e informa sobre el tipo de acceso, los formatos en que se presenta y su cobertura temporal, estableciendo un enlace con la dirección electrónica de la revista y el acceso al texto completo de los artículos disponibles. Para octubre de 2012, este directorio registra más de 4,700 títulos.

\section{Portal de Portales}

Es un macro portal, creado en 2011, que reúne y da acceso a los contenidos de una selección de revistas académicas iberoamericanas disponibles en 18 hemerotecas virtuales creadas en la región por diversas instituciones: Dialnet, e-Revistas, Lamjol, Pepsic, Racó, Redalyc, Revistas de la Universidad de Chile, Portal de Revistas Científicas y Arbitradas de la UNAM, Saber ULA, UFPR y algunos sitios nacionales de la red SciELO: Argentina, Brasil, Chile, Colombia, Cuba, España, México y Perú. Para octubre de 2012, ofrece más de 1,200,000 artículos a texto completo provenientes de más de 3,000 revistas de acceso abierto. Los portales participantes emplean el OA Harvester 2 desarrollado por el Public Knowledge Project que permite la cosecha de metadatos. Está disponible a través del sitio web de Latindex.

La coordinación general del sistema reside en el Departamento de Bibliografía Latinoamericana, Subdirección de Servicios de Información Especializada, Dirección General de Bibliotecas (DGB) de la Universidad Nacional Autónoma de México (UNAM). En cada uno de los países participantes existe una institución responsable, para Perú son el:

- Consejo Nacional de Ciencia y Tecnología (CONCYTEC)

- Centro Nacional de Documentación e Información Científica y Tecnológica (CENDICYT)

Mediante un acuerdo alcanzado en 2005, la REDIAL (Red Europea de Información y Documentación sobre América Latina) con sede en Francia, es la instancia encargada de ingresar y actualizar las revistas latinoamericanistas en el Sistema Latindex.

\section{Reconocimientos}

Diversos reconocimientos ha recibido Latindex: Premio Álvaro Pérez-Ugena a la Divulgación Científica en Comunicación, otorgado por la Universidad Rey Juan Carlos de España a través de su Facultad de Ciencias de la Comunicación y la Sociedad Latina de Comunicación Social, entregado en el III Congreso Internacional Latina de Comunicación Social, celebrado en Tenerife, España, en diciembre de 2011. El premio reconoce al grupo de trabajo de la Universidad 
Nacional Autónoma de México (UNAM) por la creación de Latindex.

En el 2005 Perú tenia 160 Revistas en el Directorio y 30 Revistas en el Catalogo, representando el $21 \%$ de Latinoamérica. Por otro lado, al mirar las Revistas agrupadas por disciplinas, Medicina representaba el 20\% de las Revistas en LATINDEX.

\section{CRITERIOS DE CALIDAD PARA REVISTAS IMPRESAS}

Los parámetros establecidos por Latindex constituyen el resultado del trabajo que ha venido desarrollando un grupo de especialistas de la región. Con base en el conocimiento que de nuestras publicaciones tienen los propios socios de Latindex, al ser profesionalmente, y desde tiempo atrás, los responsables de importantes bases de datos nacionales o regionales, y previo análisis de diversos procesos de clasificación y evaluación llevados a cabo en las últimas décadas en Iberoamérica y a nivel mundial, se elaboró una lista provisional de criterios que fue puesta a prueba mediante su aplicación a diversas muestras en cada uno de los centros cooperantes, y ajustada en función de los resultados de esta prueba piloto.

Como resultado de este ejercicio, se ha definido una lista de 33 características que son certificadas en cada caso mediante la revisión de los tres últimos fascículos publicados de la revista. La batería de características es publica y se puede consultar en la página web de Latindex. Cada uno de los 18 centros cooperantes ingresa al Catálogo los nuevos títulos correspondientes a su país, por lo que el total de títulos va creciendo conforme se avanza con el trabajo.

CREACIÓN DE UN PRODUCTO ESPECÍFICO, EL CATÁLOGO, QUE RECOGE LAS CARACTERÍSTICAS EDITORIALES DE LAS REVISTAS

El Catálogo Latindex es un producto derivado del Directorio, que vio la luz por primera vez en el año 2002 y está formado por las revistas que cumplen un mínimo de criterios de calidad. Contribuyen a él los representantes de cada país miembro conforme se van evaluando los títulos, por lo que está en constante formación.

Para que puedan ser ingresadas al Catálogo, las revistas deben cumplir con todas las características básicas y con al menos 17 de los restantes parámetros listados. Esto significa que globalmente cada revista debe cubrir al menos $75 \%$ del total de características de calidad editorial en el caso de las revistas impresas, y como mínimo $70 \%$ en el caso de las electrónicas.

Lo común en los países de la región es que el trabajo editorial no obedezca a un proceso de formación superior que provea los conocimientos para tal fin, sino que se realice como producto de la experiencia que los editores adquieren en la práctica de su ejercicio.

Por ello, como parte de sus acciones más importantes, Latindex ofrece talleres a los editores para brindar la capacitación en el campo, y logra así elevar la calidad de las revistas producidas en la región. Estos talleres se ofrecen tanto bajo la modalidad presencial como virtual, y con el apoyo de las instituciones cooperantes.

Las instituciones que realizan investigación, así como las encargadas de fomentarla o financiarla, requieren herramientas confiables para la evaluación del desempeño académico o de los resultados de la investigación. En este contexto, la evaluación de las revistas o de los artículos contenidos en ellas se ha convertido en un elemento central. Por otra parte, dado que toda evaluación implica necesariamente una comparación, para poder evaluar los elementos de un conjunto se requiere tener un conocimiento del conjunto en su totalidad.

En los países de lberoamérica era importante que tanto las instituciones de investigación como los organismos nacionales de Ciencia y Tecnología contaran con una alternativa al uso casi exclusivo que tradicionalmente se ha hecho de la información proporcionada por el "Institute for Scientific Information" (ISI) sobre un conjunto altamente selectivo y poco representativo de las revistas de la región. Esta ha sido otra de las motivaciones centrales para la construcción del Directorio Latindex, seguida de la elaboración de los parámetros de calidad editorial y la creación del Catálogo Latindex.

Las universidades de América Latina tienen un gran desafío en la Era del Conocimiento, porque deberán pasar de ser simples receptoras del conocimiento foráneo a posicionarse como productoras autónomas de avances científicos y tecnológicos. Este imperativo surge de la responsabilidad de las universidades como centros que ocupan la máxima jerarquía en la escala de generación del saber y la construcción del pensamiento crítico que la sociedad requiere. 
Del cumplimiento de esta responsabilidad depende el futuro desarrollo científico tecnológico de los pueblos de la región, a partir de sus propias culturas y tradiciones, y sus aportes futuros a la construcción de la ciencia con rostro humano.

Cuando pensamos en desarrollo científico y tecnológico, no podemos limitarnos a la generación de planes y programas de investigación, sino que debemos tomar en cuenta la importancia de la difusión científica, su acceso y su impacto en la comunidad científica internacional. Las perspectivas de desarrollo de los sistemas de difusión de la investigación científica en América Latina son amplias, y tendrán que continuar buscando mejoras en la calidad y la accesibilidad de las publicaciones. Los sistemas y redes de difusión científica desarrollados en la región de América Latina apuntan a promover la mejora de la calidad de la investigación, así como sus posibilidades de impacto a nivel global.

La universidad Ricardo Palma debe aprovechar estas oportunidades y seguir apostando a ampliar su participación en estas redes; de este modo poder reducir las desventajas del país en cuanto al acceso global y al impacto de las investigaciones científicas desarrolladas en el país, en la comunidad científica internacional. Los criterios de calidad y condiciones exigidas por las redes regionales dedicadas a la difusión científica, no exceden a las posibilidades de realización efectiva de las universidades peruanas. Los costos cada vez menores de las publicaciones abiertas impresas y digitales permiten que todas las instituciones educativas universitarias estén en condiciones contar con sus revistas o publicaciones científicas, sin más requisitos que el esfuerzo y la cooperación de su comunidad educativa.

\section{PERSPECTIVAS DE DESARROLLO PARA UN FUTURO PRÓXIMO}

Las Revistas académicas de América Latina han aspirado a alcanzar un nivel adecuado de visibilidad e impacto no sólo localmente, sino también en el ámbito internacional. Los editores no han estado solos en este esfuerzo, otros actores de la cadena de información también han desempeñado su papel. La creación de bases de datos latinoamericanas ha mejorado la comunicación y el conocimiento de las publicaciones en la región como complementos útiles a la escasa información proporcionada por fuentes internacionales. La adhesión reciente a los principios del movimiento de acceso abierto también ha mejorado potencialmente la visibilidad y el acceso a las publicaciones de la región.

Los objetivos para indexar en Latindex nuestra Revista de la Facultad de Medicina Humana de la URP, fueron incrementar la visibilidad y el uso de la revista, elevar su calidad y nivel, y lograr su reconocimiento en nuestro propio país así como internacionalmente.

Tenemos el enorme placer ahora de compartir con toda la comunidad científica, el logro de haber calificado y cumplido con los estándares necesario para ingresar a Latindex, no solo en el Directorio de Latindex, sino además ser parte del Catálogo de Revistas Indexadas.

Nuevos retos e instancias nos esperan, el futuro a largo plazo esta aún por escribirse. Trabajando en equipo, con compromiso, pasión, innovación y creatividad avanzamos hacia nuevos desafíos.

3. Urdín Caminos, Carmen; Vázquez Valero, Manuel; Román Román, Adelaida (2003). Los criterios de calidad editorial LATINDEX en el marco de la evaluación de las revistas españolas de ciencia y tecnología. Revista Española de Documentación Científica, Vol. 26, no. 1, p. 56-73. 